\title{
The Stark effect in linear potentials
}

\author{
R W Robinett ${ }^{1}$ \\ ${ }^{1}$ Department of Physics, The Pennsylvania State University, University Park, PA \\ 16802 USA \\ E-mail: rick@phys.psu.edu
}

\begin{abstract}
We examine the Stark effect (the second-order shifts in the energy spectrum due to an external constant force) for two 1-dimensional model quantum mechanical systems described by linear potentials, the so-called quantum bouncer (defined by $V(z)=F z$ for $z>0$ and $V(z)=\infty$ for $z<0$ ) and the symmetric linear potential (given by $V(z)=F|z|$ ). We show how straightforward use of the most obvious properties of the Airy function solutions and simple Taylor expansions give closed form results for the Stark shifts in both systems. These exact results are then compared to other approximation techniques, such as perturbation theory and WKB methods. These expressions add to the small number of closed-form descriptions available for the Stark effect in model quantum mechanical systems.
\end{abstract}

PACS numbers: 03.65.Ge, 03.65.Sq, 02.30.Gp 


\section{Introduction}

There are only a handful of model quantum mechanical systems which admit exact solutions, and from which information is easily extracted in closed-form expressions. The most familiar examples include the Kepler problem (Coulomb potential for the hydrogen atom), the harmonic oscillator (for vibrational states), spherical harmonics (for rotational motion), and the infinite square well. These examples all allow for a relatively complete analysis of the mathematical descriptions of the position-space wavefunctions, including closed-form expressions for normalizations, expectation values, and even matrix elements for use in applications such as sum rules and perturbation theory.

The so-called 'quantum bouncer' system, defined by the potential

$$
V(z)=\left\{\begin{array}{cc}
F z & \text { for } 0 \leq z \\
\infty & \text { for } z<0
\end{array},\right.
$$

has been a staple of the pedagogical literature, in both articles [1] - [5] and textbooks [6] 9]. It not only has solutions given by well-studied special functions (Airy functions [10]), but it can also be analyzed using a variety of approximation methods for comparison to exact results. Most importantly, however, this 'academic' problem has received renewed attention as the simplest model for the quantum states of neutrons in the Earth's gravitational field [11]. The linear potential plus infinite wall has also been used to analyze surface-Landau-level resonance data [12] where electrons are trapped between the surface potential and a weak magnetic force given by $F=e v_{F} H / c$. In addition, related recent experimental realizations of motion in such a 'bouncer' potential also include systems of atoms [13], Bose-Einstein condensates [14], and even optical analogs ('photon bouncing balls') [15]. These proverbial 'real life' applications help to promote the quantum bouncer to the small set of important physical realizations of mathematically soluble problems which are accessible to students of quantum mechanics at the undergraduate level.

A simple extension of this problem, the symmetric linear potential, given by $V(z)=F|z|$, also shares Airy function solutions. Moreover, it has the same symmetry property as systems such as the harmonic oscillator or symmetric infinite well and so can be discussed in a similar context, including using concepts such as parity. For example, all three cases can be thought of as belonging to the class of power-law potentials of the form

$$
V^{(k)}(z)=V_{0}\left|\frac{z}{a}\right|^{k}
$$

with $k=1,2$ and $\infty$ for the three systems respectively. One can then systematically explore the behavior of energy eigenvalues [16] or even higher order effects, such as the Stark shifts [17], and how they depend on the power-law exponent $(k)$, quantum number, and physical parameters, such as the mass.

The Stark effect, the response of the quantized energy levels to the addition of a constant external force, is another staple of quantum mechanics texts, with closed 
form results possible in a number of the same tractable systems mentioned above. For example, the addition of a linear potential of the form $\bar{V}(z)=\bar{F} z$ to the harmonic oscillator $(\mathrm{HO})$ can be easily accommodated with an exact solution (requiring only a simple 'complete the squares' trick), leading to a second order shift,

$$
E_{n}^{(2)}(H O)=-\frac{\bar{F}^{2}}{2 m \omega^{2}}
$$

namely a constant, lowering the energies of all of the states. For the symmetric infinite well (SIW), with walls at $x= \pm a$, methods involving inhomogeneous differential equations [18] (the so-called Dalgarno-Lewis method [19]) and perturbation theory summation approaches [20], 21] have been used to evaluate the second order shift, yielding

$$
E_{n}^{(2)}(S I W)=\frac{\bar{F}^{2} a^{2}}{12 E_{n}^{(0)}}\left[1-\frac{15}{(n+1)^{2} \pi^{2}}\right]
$$

where

$$
E_{n}^{(0)} \equiv \frac{\hbar^{2} \pi^{2}(n+1)^{2}}{8 m a^{2}} \quad \text { where } \quad n=0,1,2, \ldots
$$

define the unperturbed energies in an infinite well of width $2 a$. In contrast to the harmonic oscillator, the Stark shift here is negative only for the ground state (as required by second-order perturbation theory) but positive for all excited states, which is an interesting qualitative difference. It is in this context that we will examine the Stark shifts for the quantum bouncer and the related symmetric linear potential. Given the simple nature of the Airy function solutions (or rather the differential equation they satisfy), it's perhaps not surprising that exact expressions for the Stark shifts in both systems can also be derived, and that is the topic of this paper.

In the next section, we review the solutions of the quantum bouncer problem, focusing on providing a list of exact results needed for quantities such as normalizations, expectation values, and other matrix elements. We find that the Stark shifts are actually trivially obtained in this case. In Sec. 3, we provide the corresponding results for the more interesting case of the symmetric linear potential. For this example, we make use of the fact that the simple form of the Airy differential equation provides dramatic simplifications for the Taylor series expansion of the exact energy eigenvalue condition for the 'perturbed' case, and we find closed form expressions for the Stark shifts in this system as well, one of our main results. In both cases, we compare the exact results to WKB approximations for the Stark shifts, which have been discussed earlier in the literature [17], as well as with second-order perturbation theory. Finally we put our results into context in Sec. 4 where we discuss our conclusions. 


\section{Stark effect for the quantum bouncer}

We begin by reviewing the solutions for the quantum bouncer problem, defined by the potential in Eqn. (11). The Schrödinger equation in the region $z>0$ reduces to

$$
-\frac{\hbar^{2}}{2 m} \frac{d^{2} \psi_{n}(z)}{d z^{2}}+F z \psi_{n}(z)=E_{n} \psi_{n}(z),
$$

which can be written in the form

$$
\psi_{n}^{\prime \prime}(x)=\left(x-\beta_{n}\right) \psi_{n}(x)
$$

using the change of variable $z=\rho x$ and the definitions

$$
\rho=\left(\frac{\hbar^{2}}{2 m F}\right)^{1 / 3} \quad \text { and } \quad \beta_{n}=\frac{E_{n}}{F \rho} \equiv \frac{E_{n}}{\mathcal{E}_{0}} .
$$

The solutions of Eqn. (7) are the two linearly-independent Airy functions, $\operatorname{Ai}\left(x-\beta_{n}\right)$ and $B i\left(x-\beta_{n}\right)$. (The now standard reference on the application of Airy functions in physics is Ref. [10].) The $B i$ solution (which diverges for large positive argument) does not satisfy the boundary condition $\psi_{n}(z \rightarrow \infty)=0$ and so is excluded. The energy eigenvalues are then determined by the boundary condition imposed by the infinite wall at the origin, namely that $\psi(z=0)=A i\left(-\beta_{n}\right)$. The quantized energies are then given in terms of the zeros of the well-behaved Airy function, $A i\left(-\zeta_{n}\right)$, with $E_{n}=+\zeta_{n} \mathcal{E}_{0}$. In many other systems, the explicit manner in which the application of the boundary conditions leads to discrete energies is less than mathematically obvious. In this case, however, one simply 'slides' the single $A i(x)$ solution along the axis (via the $\beta_{n}$ shift) until one of its zeros coincides with the origin and therefore satisfies the boundary condition at the infinite wall. (We note that Eqn. (6) can be easily solved in momentum space, and then connected to the integral representation of the Airy function [9]. The energy eigenvalue conditions for this problem, and especially our systematic expansion of them, are much more easily derived directly from the position space form of the Schrödinger equation.)

Standard handbook results for the asymptotic behavior of the $A i(x)$ zeros [22] give

$$
E_{n} \sim \mathcal{E}_{0}\left[\frac{3 \pi}{2}(n-1 / 4)\right]^{2 / 3}=\mathcal{E}_{0}\left[\frac{3 \pi}{4}(2 n-1 / 2)\right]^{2 / 3}
$$

for large $n$ (where the labeling starts with $n=1$.) We note that the WKB prediction for the energy eigenvalues for this system is

$$
\int_{0}^{E_{\tilde{n}} / F} \sqrt{2 m\left(E_{\tilde{n}}-F z\right)} d z=\left(\tilde{n}+C_{L}+C_{R}\right) \hbar \pi \quad \text { with } \quad \tilde{n}=0,1,2, \ldots
$$

and the appropriate values of the matching coefficients are $C_{L}=1 / 2$ (at the left turning point, where there is an infinite wall boundary condition) and $C_{R}=1 / 4$ (for the smoother potential at the right turning point). Solving for the quantized energies gives

$$
E_{\tilde{n}}(W K B)=\left[\frac{3 \pi(\tilde{n}+3 / 4)}{2}\right]^{2 / 3} \mathcal{E}_{0}
$$

where $\tilde{n}=0,1,2, \ldots$, which agrees with the 'handbook' result in Eqn. (9) for large $\tilde{n}$. 
The wavefunctions for positive $z$ are then given by

$$
\psi_{n}(z)=N_{n} A i\left(\frac{z}{\rho}-\zeta_{n}\right)
$$

and the orthonormality properties of these solutions can be investigated very straightforwardly. For example, the normalization constant can be determined in closed form by using one of the simple identities, Eqn. (54), involving integrals Airy functions collected in the Appendix, where we find that

$$
N_{n}=\frac{1}{\sqrt{\rho} A i^{\prime}\left(-\zeta_{n}\right)} .
$$

(Gea-Banacloche [23] was the first to find this relation numerically, and soon afterwards Vallée [24] and Goodmanson [25] demonstrated it analytically. In this work, we choose $A i^{\prime}\left(-\zeta_{n}\right)$ in the normalization instead of $\left|A i^{\prime}\left(-\zeta_{n}\right)\right|$, as done in Refs. 23] - 25], in order to simplify some of the expressions below; such a choice of phase has, of course, no physical significance.) One can then just as easily confirm that $\left\langle\psi_{n} \mid \psi_{m}\right\rangle=0$ for $n \neq m$ by using Eqn. (58).

As noted by Goodmanson [25], the expectation values of the potential and kinetic energies can be evaluated by using the identities in Eqns. (55) and (57) respectively to find

$$
\begin{aligned}
\langle n|V(z)| n\rangle & =F \int_{0}^{\infty} z\left|\psi_{n}(z)\right|^{2} d z \\
& =\frac{F \rho^{2}}{\rho\left[A i^{\prime}\left(-\zeta_{n}\right)\right]^{2}} \int_{0}^{\infty} x A i\left(x-\zeta_{n}\right)^{2} d x \\
& =(F \rho)\left(\frac{2 \zeta_{n}}{3}\right)=\frac{2}{3} E_{n}
\end{aligned}
$$

and

$$
\begin{aligned}
\frac{1}{2 m}\left\langle n\left|\hat{p}^{2}\right| n\right\rangle & =\frac{\hbar^{2}}{2 m} \int_{0}^{\infty}\left|\frac{d \psi_{n}(z)}{d z}\right|^{2} d z \\
& =\left(\frac{\hbar^{2}}{2 m \rho^{2}}\right)\left[\frac{\rho}{\rho\left[A i^{\prime}\left(-\zeta_{n}\right)\right]^{2}}\right] \int_{0}^{\infty}\left[A i^{\prime}\left(x-\zeta_{n}\right)\right]^{2} d x \\
& =\left(\frac{\hbar^{2}}{2 m \rho^{2}}\right)\left(\frac{\zeta_{n}}{3}\right)=\frac{\mathcal{E}_{0} \zeta_{n}}{3}=\frac{1}{3} E_{n}
\end{aligned}
$$

where an integration by parts has been used in Eqn. (15) to bring it to the form of the integral in Eqn. (57). (One can also make direct use of the Airy differential equation, $A^{\prime \prime}=z A$, to rewrite $\left\langle n\left|\hat{p}^{2}\right| n\right\rangle$ in terms of the same integral in Eqn. (14).) These calculations confirm that both quantities are consistent with the virial theorem. For future reference, we find that the dipole matrix elements are given by

$$
\left\langle\psi_{n}|z| \psi_{k}\right\rangle=-\frac{2 \rho}{\left(\zeta_{n}-\zeta_{k}\right)^{2}}
$$

where we use Eqn. (59).

Turning now to the Stark effect, we note that the result of a perturbing potential of the form $\bar{V}(z)=\bar{F} z$ is trivially realized since the form of the solutions remain the 
same with the global substitution $\tilde{F} \rightarrow(F+\bar{F})$. The new energy eigenvalues are thus obtained from Eqn. (8) as

$$
\tilde{E}_{n}=\left(\mathcal{E}_{0} \zeta_{n}\right)\left[1+\frac{\bar{F}}{F}\right]^{2 / 3}
$$

With this simple form, the predictions for the first-, second-, and third-order energy shifts are given by a simple expansion, namely

$$
E_{n}^{(1)}=\frac{2}{3}\left(\frac{\bar{F}}{F}\right) E_{n}^{(0)}, \quad E_{n}^{(2)}=-\frac{1}{9}\left(\frac{\bar{F}}{F}\right)^{2} E_{n}^{(0)}, \quad \text { and } \quad E_{n}^{(3)}=\frac{4}{81}\left(\frac{\bar{F}}{F}\right)^{3} E_{n}^{(0)} .
$$

The first-order perturbation theory result,

$$
E_{n}^{(1)}=\left\langle\psi_{n}|\bar{V}(z)| \psi_{n}\right\rangle=\bar{F}\left\langle\psi_{n}|z| \psi_{n}\right\rangle=\frac{2}{3}\left(\frac{\bar{F}}{F}\right)\left(\mathcal{E}_{0} \zeta_{n}\right),
$$

is easily confirmed by the same integral as in Eqn. (14). The second-order energy term is given by the standard expression

$$
E_{n}^{(2)}=\sum_{k \neq n} \frac{|\langle n|\bar{V}(z)| k\rangle|^{2}}{\left(E_{n}^{(0)}-E_{k}^{(0)}\right)}
$$

so that the predicted Stark shift for the quantum bouncer is

$$
E_{n}^{(2)}=-4\left(\frac{\bar{F}}{F}\right)^{2} \mathcal{E}_{0}\left[\sum_{k \neq n} \frac{1}{\left(\zeta_{k}-\zeta_{n}\right)^{5}}\right] .
$$

It has been shown in Ref. [26] that this result (and the corresponding double summation

giving the third-order expression, $E_{n}^{(3)}$ ) provides new constraints on combinations of zeros of Airy functions, which can be easily confirmed numerically using tools such as Mathematica ${ }^{\circledR}$. This is one example where novel applications of standard quantum mechanical methods can provide new results in mathematical physics.

We also note that the WKB approximation in Eqn. (10) has the same global redefinition of $F \rightarrow \tilde{F}=F+\bar{F}$, yielding the same additional factor of $(1+\bar{F} / F)^{2 / 3}$ found in the exact result in Eqn. (17), so that the WKB approximation gives the correct large $n$ behavior of the first- and second-order shifts as well.

\section{The symmetric linear potential}

The case of the symmetric linear potential, a seemingly small variation on the quantum bouncer potential, namely

$$
V(z)=F|z|,
$$

adds a number of interesting features, most notably making the evaluation of the $\bar{F}$ dependent shifts in energy levels more challenging. But use of the simplest properties of the Airy function solutions leads to a straightforward calculational algorithm which can still give closed-form results for the energy shifts to any desired order in $\bar{F} / F$. We begin by reviewing the solutions of the symmetric linear potential. 
The energy eigenstates in this symmetric potential can be classified by parity, and the odd-parity states are automatically related to those of the 'half-well' problem (namely the quantum bouncer above) by

$$
\psi_{n}^{(-)}(z)=\left\{\begin{array}{cc}
+\psi_{n}(z) / \sqrt{2} & \text { for } z \geq 0 \\
-\psi_{n}(-z) / \sqrt{2} & \text { for } z \leq 0
\end{array}\right.
$$

where the $\psi_{n}(z)$ are given by Eqns. (12) and (13) and the normalization is modified. Henceforth, we label the energies for these states as $E_{n}^{(-)}=\zeta_{n} \mathcal{E}_{0}$.

The corresponding even states must still be of the form $\psi_{n}^{(+)}(z)=A i\left(z / \rho-\beta_{n}\right)$ (at least for $z>0$ ), but the appropriate boundary condition is now that $\psi^{\prime}(z=0)=$ $A i^{\prime}\left(-\beta_{n}\right)=0$, so the energy eigenvalues for these states are given by $E_{n}^{(+)}=\chi_{n} \mathcal{E}_{0}$, where $-\chi_{n}$ are the zeros of the derivative of $A i(x)$. Using the integral in Eqn. (54), we find that the appropriately normalized even solutions can be written in the form

$$
\psi_{n}^{(+)}(z)=\frac{1}{\sqrt{2 \rho \chi_{n}} A i\left(-\chi_{n}\right)} A i\left(\frac{|z|}{\rho}-\chi_{n}\right),
$$

once again with an analytic result for the normalization. A standard handbook 22$]$ result for the zeros of $A i^{\prime}(x)$ gives

$$
E_{n}^{(+)} \sim \mathcal{E}_{0}\left[\frac{3 \pi}{2}(n-3 / 4)\right]^{2 / 3}=\mathcal{E}_{0}\left[\frac{3 \pi}{4}((2 n-1)-1 / 2)\right]^{2 / 3} .
$$

Comparing this to Eqn. (9), we see that the energy eigenstates thus satisfy $E_{n}^{(+)}<$ $E_{n}^{(-)}<E_{n+1}^{(+)}$and interleave, as in any Sturm-Liouville problem, and alternate in parity, as for any symmetric potential. We note that the WKB prediction for the symmetric linear potential is dictated by the quantization constraint

$$
\int_{-E_{\bar{n}} / F}^{+E_{\bar{n}} / F} \sqrt{2 m\left(E_{\bar{n}}-F|z|\right)} d z=(\bar{n}+1 / 4+1 / 4) \hbar \pi \quad \text { with } \quad \bar{n}=0,1,2, \ldots
$$

since both turning points are now 'soft' $\left(C_{L}=C_{R}=1 / 4\right)$, giving

$$
E_{\bar{n}}=\left[\frac{3 \pi(\bar{n}+1 / 2)}{4}\right]^{3 / 2} \mathcal{E}_{0} .
$$

This expression agrees with Eqn. (91) (for $\bar{n}=2 n$ ) and Eqn. (25) (for $\bar{n}=2 n-1$ ), as expected.

Simple calculations then show that virial theorem results in Eqns. (14) and (15) still hold. Furthermore, the 'diagonal' dipole matrix elements are given by

$$
\left\langle\psi_{n}^{(+)}|z| \psi_{k}^{(+)}\right\rangle=0=\left\langle\psi_{n}^{(-)}|z| \psi_{k}^{(-)}\right\rangle
$$

because of parity constraints, while using the integral in Eqn. (59) we find

$$
\left\langle\psi_{n}^{(-)}|z| \psi_{k}^{(+)}\right\rangle=-\frac{2 \rho}{\sqrt{\chi_{k}}\left(\chi_{k}-\zeta_{n}\right)^{3}} .
$$

Turning now to the Stark effect, the addition of an external constant field, $\bar{V}(z)=\bar{F} z$, changes the potential asymmetrically to

$$
\tilde{V}(z)=V(z)+\bar{V}(z)= \begin{cases}+(F+\bar{F}) z & \text { for } z>0 \\ -(F-\bar{F}) z & \text { for } z<0\end{cases}
$$


but the Schrödinger equation still supports Airy function solutions in both regions. The most general solution satisfying the boundary conditions at $\pm \infty$ is now

$$
\psi_{n}(z)= \begin{cases}\alpha_{R} A i\left(+z / \rho_{R}-E_{n} /\left(F_{R} \rho_{R}\right)\right) & \text { for } z>0 \\ \alpha_{L} A i\left(-z / \rho_{L}-E_{n} /\left(F_{L} \rho_{L}\right)\right) & \text { for } z<0\end{cases}
$$

using the definitions

$$
\begin{array}{ll}
F_{R}=F(1+\Delta) & \rho_{R}=\rho(1+\Delta)^{-1 / 3} \\
F_{L}=F(1-\Delta) & \rho_{L}=\rho(1-\Delta)^{-1 / 3}
\end{array}
$$

where $\Delta \equiv \bar{F} / F$ and the $R / L$ notations refer to the $z>0$ and $z<0$ regions respectively. The boundary conditions which must now be satisfied at $z=0$ are the continuity of both $\psi_{n}(z)$ and $\psi_{n}^{\prime}(z)$ and applying these we find the eigenvalue condition

$$
\begin{aligned}
G\left(E_{n}, \Delta\right) \equiv(1+ & \Delta)^{1 / 3} A i\left(\frac{-E_{n}}{\mathcal{E}_{0}(1-\Delta)^{2 / 3}}\right) A i^{\prime}\left(\frac{-E_{n}}{\mathcal{E}_{0}(1+\Delta)^{2 / 3}}\right) \\
& +(1-\Delta)^{1 / 3} A i\left(\frac{-E_{n}}{\mathcal{E}_{0}(1+\Delta)^{2 / 3}}\right) A i^{\prime}\left(\frac{-E_{n}}{\mathcal{E}_{0}(1-\Delta)^{2 / 3}}\right)=0 .
\end{aligned}
$$

We note that in the absence of the perturbing field we have $\Delta=0$ and the eigenvalue condition reduces to

$$
G\left(E_{n}, 0\right)=A i\left(\frac{-E_{n}}{\mathcal{E}_{0}}\right) A i^{\prime}\left(\frac{-E_{n}}{\mathcal{E}_{0}}\right)=0
$$

which is satisfied by either $E_{n} / \mathcal{E}_{0}=\chi_{n}$ (giving the even states) or $\zeta_{n}$ (giving the odd states), as expected.

Assuming that $\Delta<<1$, one can expand $G\left(E_{n}, \Delta\right)$ about the unperturbed values using standard Taylor series expansions of the $A i(x)$ and $A i^{\prime}(x)$ functions. This expansion is immensely simplified by repeated use of the fact that the $A i(x)$ satisfies the Airy differential equation, namely $A i^{\prime \prime}(x)=x A i(x)$. For example, if we need

$$
A i(y+\delta)=A i(y)+\delta A i^{\prime}(y)+\frac{\delta^{2}}{2 !} A i^{\prime \prime}(y)+\frac{\delta^{3}}{3 !} A i^{\prime \prime \prime}(y)+\frac{\delta^{4}}{4 !} A i^{(i v)}(y)+\cdots
$$

we can use the relations

$$
\begin{aligned}
A i^{\prime \prime}(y) & =y A i(y) \\
A i^{\prime \prime \prime}(y) & =\left[A i^{\prime \prime}(y)\right]^{\prime}=[y A i(y)]^{\prime}=A i(y)+y A i^{\prime}(y) \\
A i^{(i v)}(y) & =\left[A i^{\prime \prime \prime}(y)\right]^{\prime}=\left[A i(y)+y A i^{\prime}(y)\right]^{\prime}=2 A i^{\prime}(y)+y A i^{\prime \prime}(y) \\
& =2 A i^{\prime}(y)+y^{2} A i(y) \\
\vdots & =
\end{aligned}
$$

Thus, the derivatives at any order can be expressed as a combination of $\operatorname{Ai}(y)$ and $A i^{\prime}(y)$ (each times polynomials in $y$ ), but no higher derivatives. Then, since we are expanding about $y=-\chi_{n}$ or $y=-\zeta_{n}$, one or the other of these terms will vanish (either $A i\left(-\zeta_{n}\right)=0$ or $A i^{\prime}\left(-\chi_{n}\right)=0$ ). These simplifications leave the expansion of $G\left(E_{n}, \Delta\right)$ proportional to the non-vanishing term squared, multiplying a polynomial in $\Delta$ with coefficients containing polynomials in $\chi_{n}$ or $\zeta_{n}$. 
So, the strategy is to write

$$
E_{n}=x \mathcal{E}_{0}\left(1+R_{1} \Delta+R_{2} \Delta^{2}+R_{3} \Delta^{3}+\cdots\right)
$$

where $x=\chi_{n}$ or $\zeta_{n}$, expand $G\left(E_{n}, \Delta\right)$ in terms of powers of $\Delta$, and make repeated use of the identities in Eqns. (37) - (40). The resulting expression in powers of $\Delta$ must vanish identically, so that its coefficients (which will contain the dimensionless $R_{i}$ factors) must vanish term-by-term.

As an example, expanding around $E_{n}=\zeta_{n} \mathcal{E}_{0}$ for the odd states (for which $\left.A i\left(-\zeta_{n}\right)=0\right)$ gives the constraint

$$
\begin{aligned}
G\left(E_{n}^{(-)}, \Delta\right)=\left[A i^{\prime}\left(-\zeta_{n}\right)\right]^{2}\{ & -2 \zeta_{n} R_{1} \Delta-\frac{\left(14+18 R_{2}\right) \zeta_{n}}{9} \Delta^{2} \\
& \left.-\frac{\left(3 R_{3}+2 R_{1}-2 R_{1}^{3} \zeta_{n}^{3}\right) \zeta_{n}}{3} \Delta^{3}+\cdots\right\}=0
\end{aligned}
$$

up to third order in $\Delta=\bar{F} / F$. We immediately see that $R_{1}=R_{3}=0$ as dictated by the symmetry of the problem and perturbation theory results. Recall that the first-order shift in Eqn. (19) vanishes in this case due to parity (since the diagonal dipole matrix elements vanish, as in Eqn. (28) ). The third-order shift, given by

$$
E_{n}^{(3)}=\sum_{k \neq n} \sum_{j \neq n} \frac{\langle n|\bar{V}(z)| k\rangle\langle k|\bar{V}(z)| j\rangle\langle j|\bar{V}(z)| n\rangle}{\left(E_{n}^{(0)}-E_{k}^{(0)}\right)\left(E_{n}^{(0)}-E_{j}^{(0)}\right)}-\langle n|\bar{V}(z)| n\rangle \sum_{k \neq n} \frac{|\langle n|\bar{V}(z)| k\rangle|^{2}}{\left(E_{n}^{(0)}-E_{k}^{(0)}\right)^{2}},
$$

can then be seen to explicitly vanish as well for similar reasons.

The new non-trivial result is that $R_{2}=-7 / 9$, so that the Stark shift for the odd states is given by

$$
E_{n}^{(-, 2)}=-\frac{7}{9}\left(\frac{\bar{F}}{F}\right)^{2}\left(\zeta_{n} \mathcal{E}_{0}\right)=-\frac{7}{9}\left(\frac{\bar{F}}{F}\right)^{2} E_{n}^{(-, 0)} .
$$

In exactly the same manner, expanding about $E_{n}=\chi_{n} \mathcal{E}_{0}$, we find the corresponding result for the Stark shift for the even states, namely

$$
E_{n}^{(+, 2)}=-\frac{5}{9}\left(\frac{\bar{F}}{F}\right)^{2}\left(\chi_{n} \mathcal{E}_{0}\right)=-\frac{5}{9}\left(\frac{\bar{F}}{F}\right)^{2} E_{n}^{(+, 0)} .
$$

If we include the perturbing potential, the WKB prediction for the energy eigenvalues is now determined by the quantization condition

$$
\sqrt{2 m} \int_{a_{-}}^{a_{+}} \sqrt{\tilde{E}_{n}-(F|z|+\bar{F} z)} d z=(n+1 / 2) \pi \hbar
$$

where $n=0,1,2, \ldots$ and the classical turning points are

$$
a_{ \pm}= \pm \frac{E_{n}}{(F \pm \bar{F})} \text {. }
$$

The resulting approximation for the energy eigenvalues is found to be

$$
\tilde{E}_{n}=E_{n}\left(1-\frac{\bar{F}^{2}}{F^{2}}\right)^{2 / 3} \approx E_{n}\left(1-\frac{2}{3} \Delta^{2}+\cdots\right)
$$


where the $E_{n}$ are given by the $\bar{F}=0 \mathrm{WKB}$ result in Eqn. (27). In this approximate approach, the first- and third-order terms (in $\Delta$ ) do indeed vanish, while the secondorder Stark shift is given by

$$
E_{n}^{(2)}=-\frac{2}{3} E_{n} \Delta^{2}=-\frac{6}{9}\left(\frac{\bar{F}}{F}\right)^{2} E_{n}^{(0)}
$$

with no dependence on parity, since that concept plays no role in the semi-classical WKB approach. (The Stark shifts in various power-law potentials have been examined in Ref. [17] using just such a WKB approach, where this last result was first noted.)

Interestingly then, we find that the exact second-order results in Eqns. (43) and (44) bracket the approximate WKB result and in fact give it as the 'average' effect, but they do depend on the parity of the unperturbed eigenstate. Both approaches give the correct dependence as being proportional to the unperturbed energy eigenvalue.

Finally, in the spirit of Ref. [26], we can use the standard second-order perturbation theory expression in Eqn. (20) and the dipole matrix elements in Eqns. (28) and (29) to find

$$
\begin{aligned}
& E_{n}^{(-, 2)}=-4\left(\frac{\bar{F}}{F}\right)^{2} \mathcal{E}_{0}\left[\sum_{k} \frac{1}{\chi_{k}\left(\chi_{k}-\zeta_{n}\right)^{7}}\right] \\
& E_{n}^{(+, 2)}=-4\left(\frac{\bar{F}}{F}\right)^{2} \mathcal{E}_{0}\left[\sum_{k} \frac{1}{\chi_{n}\left(\zeta_{k}-\chi_{n}\right)^{7}}\right]
\end{aligned}
$$

which leads to two new constraints on the $\chi_{n}, \zeta_{n}$, namely

$$
\left(\frac{1}{\chi_{n}}\right) \sum_{k} \frac{1}{\left(\zeta_{k}-\chi_{n}\right)^{7}}=+\frac{5 \chi_{n}}{36} \quad \text { and } \quad \sum_{k} \frac{1}{\chi_{k}\left(\chi_{k}-\zeta_{n}\right)^{7}}=+\frac{7 \zeta_{n}}{36}
$$

both of which are easily confirmed numerically using programs such as Mathematica ${ }^{\circledR}$. (In fact, in the spirit of Ref. [23], we actually found numerical evidence for these expressions first, and then derived them in closed form using the approach followed above.)

We note that the expansion of $G\left(E_{n}, \Delta\right)$ can be continued to essentially arbitrarily high order, by extending the Airy function identities in Eqns. (37) - (40). Comparing the next non-trivial result $\left(E_{n}^{( \pm, 4)}\right)$ to standard expressions for the 4th-order energy shift (see, e.g., Ref. [27] for the form), for example, yields a complicated constraint on the $\zeta_{n}, \chi_{n}$ in the form of a triple infinite summation.

\section{Discussion and conclusions}

We have derived closed form expressions for the second-order energy shift due to a constant external field (Stark effect) for two model systems, using simple properties of the solutions of the Schrödinger equation for the linear potential relevant for both problems. These expressions add to the handful (almost literally) of exact results for the Stark shifts which are available for mathematically tractable model systems in quantum mechanics (including the hydrogen atom, harmonic oscillator, and infinite well.) 
The results in Eqn. (43) and (44) for the symmetric linear potential are very similar, but not identical to, a simple WKB prediction, but with an explicit dependence on the parity of the state. In contrast, the exact results for the quantum bouncer are trivially implemented and agree with both the WKB approach as well as perturbation theory (up to at least 3rd order [26].)

We can now compare the results for the symmetric linear potential to two other familiar cases, such as the harmonic oscillator result in Eqn. (3) or the infinite well in Eqn. (44) where the first order results both vanish as well. The form of the second-order shift in Eqn. (20) requires that the result for $E_{n}^{(2)}$ for the ground state of any system (assumed non-degenerate) is always negative and that is indeed confirmed. Similarly to the harmonic oscillator, the second-order shifts for all higher states here are also negative, but are proportional to the initial energies, and not constant. This result is conceptually consistent with the WKB discussions in Ref. [17] and has the dependence on quantum number predicted there, but the simple WKB approach does not correctly distinguish between the results for different parity quantum solutions.

One well-known textbook author [28] in discussing second-order perturbation theory has noted that "...the response of any system to a perturbing force is to deform at the expense of that force and hence to decrease the potential energy of interaction". He concludes, however, that "...on physical grounds we expect the second-order correction to lower the energy in general but have proved this to be the case only for the ground state." Our analysis provides other examples of where this intuition is proved correct, with the explicit form derived in closed form for all quantum states in the system.

Motivated by such observations, the discussion in Ref. [17] used WKB approximations to examine the 'cross-over' behavior of power-law potentials of the form in Eqn. (2) where for small $k$ ( $k=1,2$ for the symmetric linear potential and oscillator) the second-order shifts were all negative, while for large $k(k \rightarrow \infty$ for the infinite well) all values of $E_{n}^{(2)}$ were positive, except as required for the ground state. One wanted to see just how 'stiff' the potential had to be for the large $n$ (or even $n>1$ ) behavior of the second-order shifts to change qualitatively in sign. The results derived here provide additional evidence of the validity of such a WKB approach to that general question, but also give more examples of exact closed-form expressions for the Stark effect, and how they depend on the details of the quantum solutions, in this case their parity.

In our review of the properties of the quantum bouncer and symmetric linear potential, we have collected several existing closed-form results related to the two systems, while providing a number of new analytic examples. Each of these results depend on relatively straightforward identities (such as the integrals in the Appendix) or other properties of the Airy functions, all directly related to the simple form of the differential equation they solve. Many of the 'standard' model quantum mechanical systems which are of relevance to important experiments (Coulomb potential, harmonic oscillator, spherical harmonics) require moderately sophisticated mathematical physics techniques to obtain even some of the simplest results; one exception is the infinite well, which relies only on simple integrals involving familiar trigonometric functions. 
Another often discussed problem is the Stark effect for the plane rotator [29] - 32] for which the application of perturbation theory is less than straightforward, so much so that a number of textbooks have evidently gotten it wrong [33] - 37]. The correct results in that case can also be obtained by explicit expansion of the exact eigenvalue condition, but this requires knowing the properties of Mathieu function. In contrast to these cases, the results shown here are all derivable (or can be easily checked by simple differentiation) using only the fact that $A i^{\prime \prime}(x)=x A i(x)$ and little else, making them very accessible to students of quantum mechanics at the undergraduate level, without the need for advanced mathematical background. The fact that they satisfy the Schrödinger equation is all that's needed.

Our focus on these analytic solutions is motivated by recent discussions of new mathematical results [23] - 25] involving Airy functions using just such simple identities. Many of these results have been in the research literature for decades [38], 39], but are only now finding use because of the application of this once 'abstract' problem to novel physical systems [11] - [15].

As recently as 1992, one author [5] was led to describe the analytic solutions of the

quantum bouncer in the following way: "This technique, which leads to Airy functions, tends to be algebraically tedious and not particularly instructive; it is best reserved for more important potentials, such as the harmonic oscillator or Coulomb potentials." Given the increased physical relevance of this model system, and its mathematically tractable nature, we argue both for its utility as a useful pedagogical example and for an enhanced position in the pantheon of canonical quantum mechanical showcase problems.

\section{Appendix}

We collect here some of the useful identities involving integrals over Airy functions needed in this paper. Versions of these results have been derived in Refs. [38] and [39]. In each case, the identities are most easily proved by direct differentiation of the right hand sides. (For example, Albright [39] notes that "Like most results in integral calculus, they are easier to check than to obtain.")

We first assume that $A(x)$ and $B(x)$ are any two linearly-independent solutions of the Airy differential equation,

$A(x-\beta)=a A i(x-\beta)+b B i(x-\beta) \quad$ and $\quad B(x-\beta)=c A i(x-\beta)+d B i(x-\beta)$

where

$$
A^{\prime \prime}(x)=(x-\beta) A(x-\beta) \quad \text { and } \quad B^{\prime \prime}(x-\beta)=(x-\beta) B(x-\beta)
$$

where we consider the shifted arguments needed for solutions of the quantum bouncer and symmetric linear potential. We then have the following identities involving indefinite integrals of products of the $A, B$, their derivatives, and various moments. 
Stark effect for linear potentials

The first identity below is needed for the normalization of wavefunctions, while the second and third are used to evaluate expectation values.

$$
\begin{gathered}
\begin{array}{c}
\int A(x-\beta) B(x-\beta) d x=(x-\beta) A(x-\beta) B(x-\beta)-A^{\prime}(x-\beta) B^{\prime}(x-\beta) \\
\begin{aligned}
\int x A(x-\beta) B(x-\beta) d x=\frac{1}{3}\left(x^{2}+\beta x-2 \beta^{2}\right) A(x-\beta) B(x-\beta) \\
+
\end{aligned} \\
\quad \frac{1}{6}\left\{A^{\prime}(x-\beta) B(x-\beta)+A(x-\beta) B^{\prime}(x-\beta)\right\} \\
\quad-\frac{1}{3}(x+2 \beta) A^{\prime}(x-\beta) B^{\prime}(x-\beta)
\end{array} \\
\begin{aligned}
\int x^{2} A(x-\beta) B(x-\beta) d x=\frac{1}{15}\left(3 x^{3}+\beta x^{2}+4 \beta^{2} x-8 \beta^{3}-3\right) A(x-\beta) B(x-\beta) \\
+
\end{aligned} \\
\quad \frac{1}{15}(3 x+2 \beta)\left\{A^{\prime}(x-\beta) B(x-\beta)+A(x-\beta) B^{\prime}(x-\beta)\right\} \\
\quad-\frac{1}{15}\left(3 x^{2}+4 \beta x+8 \beta^{2}\right) A^{\prime}(x-\beta) B^{\prime}(x-\beta) .
\end{gathered}
$$

The following identity involving derivatives is useful for evaluation of the expectation value of the kinetic energy operator and can be derived from one in Ref. 39] by a simple change of variables.

$$
\begin{gathered}
\int A^{\prime}(x-\beta) B^{\prime}(x-\beta) d x=\frac{1}{3}\left[\left\{A^{\prime}(x-\beta) B(x-\beta)+A(x-\beta) B^{\prime}(x-\beta)\right\}\right. \\
\left.+(x-\beta) A^{\prime}(x-\beta) B^{\prime}(x-\beta)-(x-\beta)^{2} A(x-\beta) B(x-\beta)\right] .
\end{gathered}
$$

We often need to consider integrals involving solutions with two different 'shifts' $\left(\beta_{1} \neq \beta_{2}\right)$, for example in the confirmation of the orthogonality of eigenstates (first identity) or the evaluation of dipole matrix elements, as in Eqns. (16) and (29).

$$
\begin{gathered}
\int A\left(x-\beta_{1}\right) B\left(x-\beta_{2}\right) d x=\frac{1}{\left(\beta_{2}-\beta_{1}\right)}\left\{A^{\prime}\left(x-\beta_{1}\right) B\left(x-\beta_{2}\right)\right. \\
\left.-A\left(x-\beta_{1}\right) B^{\prime}\left(x-\beta_{2}\right)\right\} \\
\int x A\left(x-\beta_{1}\right) B\left(x-\beta_{2}\right) d x=\frac{\left(\beta_{1}+\beta_{2}-2 x\right)}{\left(\beta_{1}-\beta_{2}\right)^{2}} A\left(x-\beta_{1}\right) B\left(x-\beta_{2}\right) \\
+\left\{\frac{x}{\left(\beta_{2}-\beta_{1}\right)}+\frac{2}{\left(\beta_{2}-\beta_{1}\right)^{3}}\right\}\left\{A^{\prime}\left(x-\beta_{1}\right) B\left(x-\beta_{2}\right)-A\left(x-\beta_{1}\right) B^{\prime}\left(x-\beta_{2}\right)\right\} \\
\frac{2}{\left(\beta_{1}-\beta_{2}\right)^{2}} A^{\prime}\left(x-\beta_{1}\right) B^{\prime}\left(x-\beta_{2}\right) .
\end{gathered}
$$

[1] Langhoff P W 1971 Schrödinger particle in a gravitational well Am. J. Phys. 39 954-7

[2] Gibbs R L 1975 The quantum bouncer Am. J. Phys. 43 25-8

[3] Desko R D and Bord D. J 1983 The quantum bouncer revisited Am. J. Phys. 51 82-4

[4] Goodings D A and Szeredi T 1991 The quantum bouncer by path integral method Am. J. Phys. 59 924-930 
[5] Whineray S 1992 An energy representation approach to the quantum bouncer Am. J. Phys 60 948-50

[6] Winter R 1986 Quantum Physics Second Edition (Davis: Faculty Publishing) pp 92-96

[7] Morrison M 1990 Understanding Quantum Physics: A User's Manual (Englewood Cliffs, NJ; Prentice-Hall) pp 413-420

[8] Sakurai J J (Tuan S F Editor) Modern Quantum Mechanics: Revised Edition (Reading, MA: Addison-Wesley) pp 108-109

[9] Goswami A Quantum Mechanics (Dubuque, IA: Wm. C. Brown) pp 88-91

[10] Vallée O and Soares M 2004 Airy Functions and Applications to Physics (New Jersey: World Scientific)

[11] Nesvizhevsky V V et al. 2002 Quantum states of neutrons in the Earth's gravitational field Nature 415 297-9; 2003 Measurement of quantum states of neutrons in the Earth's gravitational field Phys. Rev. D 67102002 (9 pages)

[12] Wanner M, Doezema R E and Strom U Far-infrared surface-Landau-level spectroscopy in Bi Phys. Rev. B 12 2883-2982

[13] Aminoff C G et al. 1993 Cesium atoms bouncing in a stable gravitational cavity Phys. Rev.. Lett. 71 3083-3086

[14] Bongs K et al. 1999 Coherent evolution of bouncing Bose-Einstein condensates Phys. Rev. Lett. 83 3577-3580

[15] Della Valle G et al. 2009 Experimental observation of a photon bouncing ball Phys. Rev. Lett. 102 180402 (4 pages)

[16] Sukhatme U 1973 WKB energy levels for a class of one-dimensional potentials Am. J. Phys. 41 $1015-6$

[17] Robinett R W 1998 The polarizability of a particle in power-law potentials: A WKB analysis Eur. J. Phys. 19 31-9

[18] Mavromatis H A 1991 The Dalgarno-Lewis summation technique: Some comments and examples Am. J. Phys 59 738-44

[19] Dalgarno A and Lewis J T 1955 The exact calculation of long-range forces between atoms by perturbation theory Proc. R. Soc. A 233 70-4

[20] Mavromatis H A 1993 New summation expressions obtained by combining perturbation theory formalisms, Int. J. Comp. Math 50 119-23

[21] Mavromatis H A 1993 Sum rules, as a tool for obtaining mathematical series Int. J. Math. Educ. Sci. Tech. 26 267-313

[22] Abramowitz M and Stegun I A (1965) Handbook of Mathematical Functions (Washington, D. C.: National Bureau of Standards, U. S. Printing Office) p. 450

[23] Gea-Banacloche J 1999 A quantum bouncing ball Am. J. Phys. 67 776-82

[24] Vallée O 2000 Comment on 'A quantum bouncing ball' by Julio Gea-Banacloche Am. J. Phys. 68 $672-3$

[25] Goodmanson D M 2000 A recursion relation for matrix elements of the quantum bouncer. Comment on 'A quantum bouncing ball' by Julio Gea-Banacloche Am. J. Phys. 68 866-8

[26] Belloni M and Robinett R W 2009 Constraints on Airy function zeros from quantum mechanical sum rules J. Phys. A: Math. Theor. 42, 075203 (11 pages)

[27] Hirschfelder J O, Brown W B, and Epstein, S T 1964 in Advances in Quantum Chemistry, Vol. 1 ed. by P.-O. Löwdin (New York: Academic Press) 255-268.

[28] Saxon D S 1968 Elementary quantum mechanics (New York: McGraw-Hill) pp 194-4

[29] Schwartz M and Martin M 1958 Plane rotator in a Stark field Am. J. Phys. 26 649-40

[30] Cronin J A, Greenberg D F, and Telegdi V L 1967 University of Chicago graduate problems in physics with solutions (Reading: Addison-Wesley) pp 31, 162-3

[31] Johnston G L and Sposito G 1976 On the Stark effect of the plane rotator Am. J. Phys. 44 723-8

[32] Kiang D 1978 Pedagogical aspects of a plane rotator Am. J. Phys. 46 1188-9

[33] Pauling L and Wilson E B 1935 Introduction to quantum mechanics with applications to chemistry 
(New York: McGraw-Hill) pp 177-8

[34] Kogan V I and Galitskiy V M 1963 Problems in quantum mechanics (Englewood Cliffs: PrenticeHall) pp 14, 185-6

[35] Constantinescu F and Magyari E 1971 Problems in quantum mechanics (Oxford: Pergamon Press) pp 205, 209-11

[36] Flügge S 1971 Practical quantum mechanics I (Berlin: Springer-Verlag) pp 110-2

[37] Cahn S B, Mahan G D, and Nadgorny B E 1997 A guide to physics problems, Part 2 (New York: Plenum) pp 66-7, 289-90

[38] Gordon R G 1969 New method for constructing wavefunctions for bound states and scattering $J$. Chem. Phys. 51 14-25 Appendix B

[39] Albright J R 1977 Integrals of products of Airy functions J. Phys. A: Math. Gen. 10 485-90 